\title{
A First-Principles Approach to Insulators in Finite Electric Fields
}

\author{
Ivo Souza, Jorge Íñiguez, and David Vanderbilt \\ Department of Physics and Astronomy, Rutgers University, Piscataway, New Jersey 08854-8019
}

(Dated: May 29, 2002)

\begin{abstract}
We describe a method for computing the response of an insulator to a static, homogeneous electric field. It consists of iteratively minimizing an electric enthalpy functional expressed in terms of occupied Bloch-like states on a uniform grid of $k$ points. The functional has equivalent local minima below a critical field $\mathcal{E}_{\mathrm{c}}$ that depends inversely on the density of $k$ points; the disappearance of the minima at $\mathcal{E}_{\mathrm{c}}$ signals the onset of Zener breakdown. We illustrate the procedure by computing the piezoelectric and nonlinear dielectric susceptibility tensors of III-V semiconductors.

PACS numbers: PACS: 77.22.Ch, 78.20.Bh, 42.70.Mp
\end{abstract}

The response of insulators and semiconductors to external electric fields is of fundamental as well as practical interest. It determines their dielectric, piezoelectric, and ferroelectric behavior. Much current technological interest is focused on the use of static fields to tune properties such as the dielectric function in the RF and microwave region or the index of refraction in the optical region. Although a sophisticated physical understanding of electric field effects has emerged [1, 2, 3], for the most part this has not translated into tractable computational schemes applicable to periodic solids. Efficient first-principles methods for computing derivatives of the total energy of solids with respect to a macroscopic field $\mathcal{E}$ at $\mathcal{E}=0$ do exist [ [, 5, 6, 7]. While for many applications such perturbation approaches are adequate, their extension to nonlinear order is awkward, and for some studies (e.g., field-induced structural phase transitions [8]) it is essential to perform calculations directly at finite fields.

The main difficulty is the nature of the scalar potential " $-\mathcal{E} \cdot \mathbf{r}$ ", which is nonperiodic and unbounded from below. The fact that it destroys the periodicity of the crystal potential means that methods based on Bloch's theorem do not apply. Moreover, as a result of the unbounded nature of the perturbation, the energy can always be lowered by transferring charge from the valence states in one region to conduction states in a distant region. This is the intrinsic dielectric breakdown caused by interband (or Zener) tunneling [1, 2, 3, 9]. In many practical situations Zener tunneling is negligible on the relevant time scale, and for relatively small fields the system remains in a polarized long-lived resonant state. This is the state we would like to describe. However, the absence of a welldefined ground state invalidates the variational principle that underlies the usual time-independent electronicstructure methods and leads to problematic "runaway" solutions in implementations of such approaches [10].

The few first-principles methods that have been proposed for dealing with finite fields in solids have had limited success. The supercell "sawtooth" approach [11] becomes prohibitively expensive for all but the simplest systems. A significant advance, rooted on the "modern theory of polarization" [12], was the development of a realspace method based on truncated field-polarized Wannier functions [13], which removed the need for supercells; however, the full first-principles implementation [14] was hindered by convergence problems and proved too cumbersome to find widespread use.

In this Letter we propose an alternative variational approach. It is based on the minimization of an electric enthalpy functional $\mathcal{F}$ with respect to Bloch-like functions, where $\mathcal{F}$ is comprised of the usual Kohn-Sham energy $E_{\mathrm{KS}}$ and a field coupling term $-\mathbf{P}_{\mathrm{mac}} \cdot \mathcal{E}$. Here $E_{\mathrm{KS}}$ and the polarization $\mathbf{P}_{\text {mac }}$ are expressed in terms of a set of field-polarized Bloch functions, the latter via the Berryphase theory of polarization [12]. Although for $\mathcal{E} \neq 0$ the Bloch functions are not eigenstates, they form an appropriate representation of the electronic system. We justify this approach, showing that a suitable choice of Brillouin zone sampling prevents runaway solutions. We demonstrate its implementation into a standard electronic band structure program by computing piezoelectric and linear and nonlinear dielectric properties of III-V semiconductors, finding good agreement with experiment. Our method opens up new possibilities for first-principles investigation of electric-field effects in condensed matter.

We solve for field-polarized Bloch functions $\psi_{n \mathbf{k}}(\mathbf{r})=$ $e^{i \mathbf{k} \cdot \mathbf{r}} u_{n \mathbf{k}}(\mathbf{r})$ [where $u_{n \mathbf{k}}(\mathbf{r})=u_{n \mathbf{k}}(\mathbf{r}+\mathbf{R})$ ] by minimizing the electric enthalpy functional introduced in Ref. [7],

$$
\mathcal{F}\left[u_{n \mathbf{k}} ; \mathcal{E}\right]=E_{\mathrm{KS}}-\Omega \mathbf{P}_{\mathrm{mac}} \cdot \mathcal{E},
$$

where $\Omega$ is the primitive cell volume and $\mathbf{P}_{\text {mac }}=\mathbf{P}_{\text {ion }}+$ $\mathbf{P}_{\mathrm{el}}$ is the macroscopic polarization. In a continuous- $k$ formulation, $\mathbf{P}_{\mathrm{el}}$ is $-f e /(2 \pi)^{3}$ times the sum of valenceband Berry phases 12] $\int_{\mathrm{BZ}} d \mathbf{k}\left\langle u_{n \mathbf{k}}\left|i \nabla_{\mathbf{k}}\right| u_{n \mathbf{k}}\right\rangle$ ( $f$ is the spin degeneracy and $e>0$ ). However, as we show below, it is essential to use a formulation in terms of a mesh of $N_{k}=N_{1} \times N_{2} \times N_{3} k$ points in the Brillouin zone (BZ). Then $E_{\mathrm{KS}}=\left(f / N_{k}\right) \sum_{n j}\left\langle u_{n \mathbf{k}_{j}}\left|\hat{H}_{\mathrm{KS}}\left(\mathbf{k}_{j}\right)\right| u_{n \mathbf{k}_{j}}\right\rangle$ and $\mathbf{P}_{\mathrm{el}} \cdot \mathbf{b}_{i}=(f e / \Omega) \varphi_{\mathrm{el}}^{(i)}$ where

$$
\varphi_{\mathrm{el}}^{(i)}=\frac{1}{N_{\perp}^{(i)}} \sum_{l=1}^{N_{\perp}^{(i)}} \operatorname{Im} \ln \prod_{j=0}^{N_{i}-1} \operatorname{det} S\left(\mathbf{k}_{j}^{(i)}, \mathbf{k}_{j+1}^{(i)}\right)
$$

is the string-averaged discretized Berry phase along the direction of primitive reciprocal lattice vector $\mathbf{b}_{i}$ [12. Here $S_{n m}\left(\mathbf{k}, \mathbf{k}^{\prime}\right)=\left\langle u_{n \mathbf{k}} \mid u_{m \mathbf{k}^{\prime}}\right\rangle, n$ and $m$ run over the 
$M$ occupied bands, and $N_{\perp}^{(1)}=N_{2} \times N_{3}$ is the number of strings along $\mathbf{b}_{1}$, each containing $N_{1}$ points $\mathbf{k}_{j}^{(1)}=$ $\mathbf{k}_{\perp}^{(l)}+\left(j / N_{1}\right) \mathbf{b}_{1}$.

It is implicit in this formulation that when $\mathcal{E} \neq 0$ the electronic structure can continue to be represented in terms of field-polarized Bloch-like functions $\psi_{n \mathbf{k}}$, even though they are no longer eigenstates of the Hamiltonian. It is well known that when describing an occupied subspace, one has the freedom to carry out an arbitrary unitary transformation among the states used to represent it. In this spirit, we assume that the density matrix can be written as $\rho\left(\mathbf{r}, \mathbf{r}^{\prime}\right)=\left(1 / N_{k}\right) \sum_{n \mathbf{k}} \psi_{n \mathbf{k}}^{*}\left(\mathbf{r}^{\prime}\right) \psi_{n \mathbf{k}}(\mathbf{r})$, where $n$ runs over the same number $M$ of Bloch-like states at all $\mathbf{k}$. Then $\rho\left(\mathbf{r}, \mathbf{r}^{\prime}\right)=\rho\left(\mathbf{r}+\mathbf{R}, \mathbf{r}^{\prime}+\mathbf{R}\right)$ and it follows that the charge density and other local quantities are periodic under translation by a lattice vector $\mathbf{R}$. These are familiar properties of an insulating ground state, and they turn out to hold for the field-polarized state as well, since: (i) If one starts with an insulating ground state and applies a homogeneous electric field with arbitrary time dependence, the occupied manifold preserves the above "insulating-like" properties at later times. (ii) A state that minimizes $\mathcal{F}$ is a stationary solution to the time-dependent Schrödinger equation in the presence of a static field; since it can be obtained by adiabatically turning on the field, it is guaranteed by (i) to have the above "insulating-like" properties. Proofs of (i-ii) are not difficult; details will be given elsewhere [15].

Usually, the transition to the discrete $k$ space is introduced for merely computational reasons. Here, on the contrary, the discrete $k$ formulation is required to eliminate the possibility of runaway solutions, i.e., to allow for stable stationary solutions to exist, unaffected by Zener charge-leakage. This is consistent with previous work showing that in the continuous- $k$ limit there are no stationary solutions to the static electric field problem (for an overview, see Sec. II. D of Ref. [3]). To understand why the discretization procedure endows $\mathcal{F}$ with minima, it is useful to think of it as "bending" space into a finite ring: a uniform mesh of $N_{1} \times N_{2} \times N_{3} k$ points amounts to imposing periodic boundary conditions - which have a ring topology - over a supercell of dimensions $L_{i}=N_{i} a_{i}$ $(i=1,2,3)$. For a given $k$-point mesh, $\mathcal{F}$ will have minima only if $\mathcal{E}$ is small enough that Zener tunneling is effectively suppressed. This should happen as long as the distance across which the electrons would have to tunnel in order to lower their energy is larger than the ring perimeter $L_{i}=N_{i} a_{i}$. By thinking of the field as spatially tilting the energy bands, one arrives at the condition $\mathcal{E} \cdot \mathbf{a}_{i}<\mathcal{E}_{\mathrm{c}} \cdot \mathbf{a}_{i}$ where $e \mathcal{E}_{\mathrm{c}} \cdot \mathbf{a}_{i} \simeq E_{\text {gap }} / N_{i}$ and $E_{\text {gap }}$ is a representative energy gap. We have confirmed this behavior in a one-dimensional three-band tight-binding model [13] by studying the stability of the field-polarized solutions and by checking that, for a given $k$ point mesh, $E_{\text {gap }} / e a N_{k}$ is a reasonably good estimate of $\mathcal{E}_{c}$.

The polarized state below $\mathcal{E}_{c}$ has additional insulatinglike properties, namely the absence of a steady-state cur- rent and the localization of the Wannier functions to a small portion of the ring [16, 17]. This state is related to the zero-field ground state by a continuous "deformation." Such "polarized manifolds" have been discussed in the literature [1 , 2, 3] for infinite crystals, where they were characterized as long-lived resonances. Instead, for our finite ring the state obtained by minimizing $\mathcal{F}$ is truly stationary, as discussed above.

By virtue of the nature of the Berry phase term, the functional $\mathcal{F}$ cannot be recast as the expectation value of a Hermitian operator. Because that term contains overlaps between the states at neighboring $k$ points, even in a tight-binding model without charge self-consistency, the problem must be solved self-consistently among all $k$ points. This breakdown of Bloch's theorem is the price to pay for handling, within periodic boundary conditions, a field whose scalar potential breaks translational invariance. Indeed, the Berry-phase term in $\mathcal{F}$ is the proper replacement of the usual scalar potential term $e \mathcal{E} \cdot\langle\mathbf{r}\rangle$ when using a ring topology. (Alternatively, one can switch to a vector potential formalism, which restores translational invariance to the Hamiltonian at the expense of rendering the static field problem time-dependent [18].)

Let us now describe the minimization algorithm. We have chosen an iterative "band-by-band" conjugategradients method [19] in which each occupied state $u_{n \mathbf{k}}$ is updated in sequence, although other schemes may be equally suitable. The many-electron state of interest violates inversion symmetry but not time-reversal symmetry; the latter can be used, together with any $\mathcal{E}$ preserving point-group operations, in reducing the BZ. The few differences with respect to a normal ground-state calculation stem from the $-\Omega \mathbf{P}_{\text {mac }} \cdot \mathcal{E}$ term, as follows. The gradient $\left|G_{n \mathbf{k}}\right\rangle=\delta \mathcal{F} / \delta\left\langle u_{n \mathbf{k}}\right|$ becomes

$$
\begin{gathered}
\left|G_{n \mathbf{k}}\right\rangle=\frac{f}{N_{k}} \hat{H}_{\mathrm{KS}}(\mathbf{k})\left|u_{n \mathbf{k}}\right\rangle+\frac{i f e}{4 \pi} \sum_{i=1}^{3} \frac{\mathcal{E} \cdot \mathbf{a}_{i}}{N_{\perp}^{(i)}} \times \\
\sum_{m=1}^{M}\left[\left|u_{m, \mathbf{k}_{+}^{(i)}}\right\rangle S_{m n}^{-1}\left(\mathbf{k}, \mathbf{k}_{+}^{(i)}\right)-\left|u_{m, \mathbf{k}_{-}^{(i)}}\right\rangle S_{m n}^{-1}\left(\mathbf{k}, \mathbf{k}_{-}^{(i)}\right)\right]
\end{gathered}
$$

where $\mathbf{k}_{ \pm}^{(i)}=\mathbf{k} \pm \mathbf{b}_{i} / N_{i}$ and use was made of Eq. (88) of Ref. [7]. By standard manipulations [19] this is converted into a preconditioned conjugate-gradients search direction $\left|D_{n \mathbf{k}}\right\rangle$ orthonormalized to the occupied manifold at k. The trial updated state is $\left|\widetilde{u}_{n \mathbf{k}}\right\rangle(\theta)=\cos \theta\left|u_{n \mathbf{k}}\right\rangle+$ $\sin \theta\left|D_{n \mathbf{k}}\right\rangle$. We search $\mathcal{F}(\theta)$ for a minimum, replace $\left|u_{n \mathbf{k}}\right\rangle$ by $\left|\widetilde{u}_{n \mathbf{k}}\right\rangle$, and go on to the next band.

However, the behavior of $\mathcal{F}(\theta)$ is unconventional, as is illustrated in Fig. 11. The $E_{\mathrm{KS}}(\theta)$ contribution (dashed line in Fig. A 1 is the usual one; it is periodic with period $\pi$ and has an amplitude proportional to $E_{\text {gap }}^{(\mathbf{k})} / N_{k}$. However, $-\Omega \mathbf{P}_{\text {mac }}(\theta) \cdot \mathcal{E}$ (dotted line) has a secular component arising from the fact that $\mathbf{P}_{\text {mac }}$ changes by $1 / N_{\perp}^{(i)}$ times a "quantum of polarization" $\Delta \mathbf{P}=f e \mathbf{R} / \Omega[12]$, where $\mathbf{R}$ is a (usually nonzero) lattice vector, as $\theta \rightarrow \overrightarrow{\theta+\pi}$. To understand this, consider one phase $\beta=\operatorname{Im} \ln \operatorname{det} S\left(\mathbf{k}, \mathbf{k}^{\prime}\right)$ 


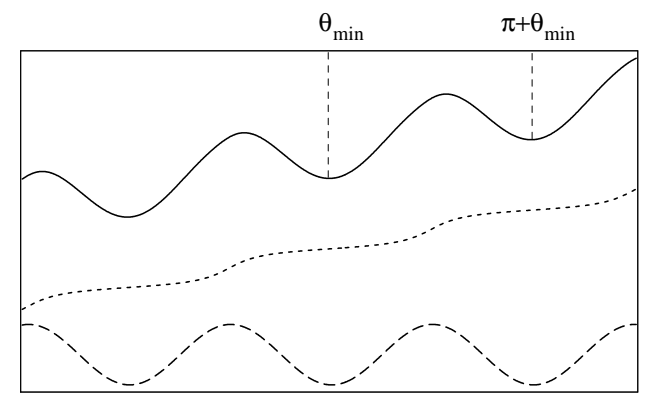

FIG. 1: The electric enthalpy $\mathcal{F}$ (solid line) and its components $E_{\mathrm{KS}}$ (dashed line) and $-\Omega \mathbf{P}_{\mathrm{mac}} \cdot \mathcal{E}$ (dotted line), plotted as a function of the parameter $\theta$ that controls the update of a polarized Bloch state in a conjugate-gradients step.

contributing to Eq. (2). Its $\theta$-dependence is $\beta(\theta)=$ $\operatorname{Im} \ln (A \cos \theta+B \sin \theta)$, where $A=\operatorname{det} S$ and $B=\operatorname{det} \widetilde{S}$ are complex constants and $\widetilde{S}$ is obtained from $S$ by replacing the $n$-th row with $\left\langle D_{n \mathbf{k}} \mid u_{m \mathbf{k}^{\prime}}\right\rangle$. It is then easy to see that $\beta(\theta)$ progresses by $\pm \pi$ as $\theta$ increases by $\pi$ (the sign depending on the sign of $\operatorname{Im} A^{*} B$ ). In total there are six such contributions for each $\mathbf{k}$; for $\mathcal{E}$ along $\mathbf{b}_{i}$, the two involving neighboring $\mathbf{k}^{\prime}$ along the string direction $i$ contribute to an average slope $-f e \mathcal{E} \cdot \mathbf{a}_{i} / N_{\perp}^{(i)} \pi$ of $-\Omega \mathbf{P}_{\mathrm{mac}}(\theta) \cdot \mathcal{E}$ as a function of $\theta$, as shown by the dotted line in Fig. 1. If $\mathcal{E}$ is not too large, we simply carry out the update by stepping to the nearest local minimum of $\mathcal{F}(\theta)$. (Local minima separated by $\pi$ are equivalent.) However, $\mathcal{F}(\theta)$ loses its minima when $\mathcal{E}$ gets too big. This occurs for $e \mathcal{E} \cdot \mathbf{a}_{i} \simeq E_{\text {gap }}^{(\mathbf{k})} / N_{i}$, which is precisely the heuristic condition for the onset of Zener tunneling on a ring.

One possible concern with the present method is that it imposes a minimum mesh spacing that can be used for a given $\mathcal{E}$. This difficulty can be circumvented in practice by decomposing the fine $k$ mesh into a set of sparser submeshes, computing the Berry-phase terms on each submesh, and then averaging over all of them. Of course, $E_{\mathrm{KS}}$ can be computed on the fine mesh.

We now turn to the computation of forces and stresses at $\mathcal{E} \neq 0$. According to the Hellmann-Feynman argument [19], at a stationary point of $\mathcal{F}$ the force $\mathbf{F}_{j} \equiv$ $-d \mathcal{F} / d \mathbf{r}_{j}$ becomes simply $-\partial \mathcal{F} / \partial \mathbf{r}_{j}$, i.e., the implicit dependence via the wave functions can be dropped. Eq. (2) has no such explicit dependence, and so does not contribute to $\mathbf{F}_{j}$. Thus, aside from the trivial ionic core contribution $e Z_{j} \mathcal{E}$, the force is given by the standard $\mathcal{E}=0$ Hellmann-Feynman expression arising from $E_{\mathrm{KS}}$ alone.

As for the macroscopic stress, similar arguments yield $\sigma_{\alpha \beta}=(1 / \Omega) \partial \mathcal{F} / \partial \eta_{\alpha \beta}$, where $\boldsymbol{\eta}$ is a homogeneous strain. However, the electric boundary conditions under which the strain derivative is taken must be specified carefully. When the cell is deformed according to $\mathbf{a}_{i} \rightarrow(\mathbf{1}+\boldsymbol{\eta}) \mathbf{a}_{i}$, we can hold fixed either the macroscopic field $\mathcal{E}$, or the potential drop across each lattice vector, $V_{i}=-\mathcal{E} \cdot \mathbf{a}_{i}$. As the Berry phases $\varphi_{\mathrm{el}}^{(i)}$ do not depend explicitly on the strain, it follows from the expression

$$
2 \pi \mathbf{P}_{\mathrm{el}} \cdot \mathcal{E}=\sum_{i=1}^{3}\left(\mathcal{E} \cdot \mathbf{a}_{i}\right)\left(\mathbf{P}_{\mathrm{el}} \cdot \mathbf{b}_{i}\right)=\frac{f e}{\Omega} \sum_{i=1}^{3}\left(\mathcal{E} \cdot \mathbf{a}_{i}\right) \varphi_{\mathrm{el}}^{(i)}
$$

that $\partial\left(\Omega \mathbf{P}_{\mathrm{el}} \cdot \mathcal{E}\right) / \partial \eta_{\alpha \beta}=0$ when $\mathbf{V}$ is fixed (the same holds true for the ionic term, which can also be recast in terms of a phase $\varphi_{\text {ion }}^{(i)}$ [20]). As a result, the stresses in the two cases are related by

$$
\sigma_{\alpha \beta}^{(\mathcal{E})}=\sigma_{\alpha \beta}^{(\mathbf{V})}-\frac{f e}{2 \pi \Omega} \sum_{i=1}^{3} \mathcal{E}_{\alpha}\left(a_{i}\right)_{\beta}\left(\varphi_{\mathrm{el}}^{(i)}+\varphi_{\mathrm{ion}}^{(i)}\right),
$$

so that the pressures differ by $\left(\mathbf{P}_{\mathrm{mac}} \cdot \mathcal{E}\right) / 3$. The stress $\boldsymbol{\sigma}^{(\mathbf{V})}$ is given in terms of the polarized Bloch states by the same expression as the stress in a zero-field ground-state calculation; it is a symmetric (torque-free) bulk quantity. On the contrary, $\boldsymbol{\sigma}^{(\mathcal{E})}$ generally has an antisymmetric part, and moreover it depends on the choice of branch cut when evaluating the multivalued bulk polarization. (In the context of a finite crystallite, the torque and extra stress in $\boldsymbol{\sigma}^{(\mathcal{E})}$ can be regarded as arising from forces exerted on the polarization-induced surface charges by a field that is held fixed in the "laboratory frame" [21].) It is straightforward to show that $c_{\alpha \beta \gamma}^{(\mathcal{E})}=d \sigma_{\beta \gamma}^{(\mathcal{E})} / d \mathcal{E}_{\alpha}$ is the so-called "improper" piezoelectric tensor [20], whereas

$$
c_{\alpha \beta \gamma}^{(\mathbf{V})}=\frac{d \sigma_{\beta \gamma}^{(\mathbf{V})}}{d \mathcal{E}_{\alpha}}=-\sum_{i=1}^{3} \frac{d \sigma_{\beta \gamma}^{(\mathbf{V})}}{d V_{i}} a_{i, \alpha}
$$

is the "proper" tensor.

The scheme outlined above was first validated on a onedimensional tight-binding model [13], where the results were found to agree with the results of the Wannier-based approach [13]. It was then implemented in ABINIT [22, a fully self-consistent pseudopotential code. To illustrate the utility of the method, we have calculated by finitedifferences dielectric and piezoelectric constants of several III-V semiconductors. That is, we increase $\mathcal{E}$ in small increments and compute the changes in the resulting forces, stresses, and polarizations, with internal displacements and strain either clamped or unclamped as appropriate. The Born effective charge is $e Z_{j \alpha \beta}^{*}=$ $d F_{j \beta} / d \mathcal{E}_{\alpha}$. (Contrary to previous finite-difference approaches, we compute it as the derivative of a force with respect to $\mathcal{E}$, not polarization with respect to displacement.) The dielectric constant is $\epsilon_{\alpha \beta}=\delta_{\alpha \beta}+\chi_{\alpha \beta}$, where $\chi_{\alpha \beta}=\left(1 / \epsilon_{0}\right) d\left(P_{\mathrm{mac}}\right)_{\alpha} / d \mathcal{E}_{\beta}$ and $\epsilon_{0}$ is the vacuum permittivity. If the ions are kept fixed, this yields the electronic contribution $\epsilon_{\infty}$; if both electrons and ions are allowed to relax in response to the field, the static dielectric constant $\boldsymbol{\epsilon}_{\text {stat }}$ is obtained. The quadratic susceptibility is $\chi_{\alpha \beta \gamma}^{(2)}=\left(2 / \epsilon_{0}\right) d^{2}\left(P_{\text {mac }}\right)_{\alpha} / d \mathcal{E}_{\beta} d \mathcal{E}_{\gamma}$, and we have computed it keeping the ions fixed. In the zinc blende structure, the only nonzero independent components of these tensors 


\begin{tabular}{lcccc}
\hline \hline & GaAs & AlAs & GaP & AlP \\
\hline a (a.u.) & 10.45 & 10.59 & 10.11 & 10.24 \\
& $(10.68)$ & $(10.69)$ & $(10.28)$ & $(10.33)$ \\
$Z_{\text {cation }}^{*}$ & 2.00 & 2.14 & 2.10 & 2.24 \\
& $(2.07)$ & $(2.18)$ & $(2.04)$ & $(2.28)$ \\
$\epsilon_{\infty}$ & 11.9 & 9.6 & 9.4 & 8.1 \\
& $(10.9)$ & $(8.2)$ & $(9.0)$ & $(7.5)$ \\
$\epsilon_{\text {static }}$ & 13.5 & 11.5 & 11.2 & 10.2 \\
& $(13.2)$ & $(10.1)$ & $(11.1)$ & $(9.8)$ \\
$\chi^{(2)}(\mathrm{pm} / \mathrm{V})$ & 134 & 64 & 66 & 39 \\
& $(166)$ & & $(74)$ & \\
$\left(a^{2} / e\right) \gamma_{14}$ & -0.40 & -0.10 & -0.25 & 0.05 \\
$\left(a^{2} / e\right) \gamma_{14}^{(0)}$ & $(-0.32)$ & & $(-0.18)$ & \\
\hline \hline
\end{tabular}

TABLE I: Computed dielectric and piezoelectric properties of III-V semiconductors. Parentheses denote experimental data quoted in Refs. $4,5,23,24$.

are $Z_{11}^{*}, \epsilon_{11}$, and $\chi_{123}^{(2)}$. The "proper" piezoelectric coefficient $c_{123}^{(\mathbf{V})}$ is computed using Eq. (6), with both clamped $\left(\gamma_{14}^{(0)}\right)$ and unclamped $\left(\gamma_{14}\right)$ ions.

The calculations were performed at the theoretical lattice constant $a$ using an energy cutoff of $10 \mathrm{Ha}$. We checked that our $k$ point sampling was very well converged at $16 \times 16 \times 16$ points in the full BZ. With this mesh spacing we find critical fields of the order of $10^{7} \mathrm{~V} / \mathrm{cm}$, and the finite-difference field step size was approximately $1 / 10$ of this value. We checked that our values for $Z^{*}$, $\gamma_{14}$, and $\gamma_{14}^{(0)}$ essentially coincide, for any given mesh of $k$ points, with those computed using the approach of Refs. [12, 20]. We have also computed $Z^{*}$ and $\epsilon_{\infty}$ by treating the electric field via density-functional perturbation theory (DFPT) [4. In the limit of a dense mesh the two approaches yield the same results; the discrepancies that occur for sparser meshes can be attributed to the different ways in which derivatives with respect to $\mathbf{k}$ are handled in each case 80. Our results for the piezoelectric coefficients and for $\chi^{(2)}$ are also in good agreement with experiment and with previous calculations using different methods [4, 5, 12].

All of the quantities reported in Table I could have been obtained using DFPT methods. However, a considerable gain in convenience is afforded by computing them using simple finite differences of $\mathcal{E}$. For example, the calculation of $\chi^{(2)}$ by DFPT is quite tedious and requires a special-purpose program, while $\chi^{(n)}$ of arbitrary order are easily computed using the present approach.

To summarize, we have presented a practical firstprinciples scheme for computing the electronic structure of insulators under a finite dc bias. The algorithm is ideally suited for implementation in a standard electronic structure code and its computational cost is comparable. Dielectric polarization, forces and stresses are straightforwardly obtained as byproducts of the calculation. The extension of this approach to time-dependent fields will be discussed in a future communication.

This work was supported by NSF Grant DMR9981193. We thank Ralph Gebauer for stimulating discussions.
[1] E. O. Kane, J. Phys. Chem. Solids 12, 181 (1959).

[2] G. H. Wannier, Phys. Rev. 117, 432 (1960).

[3] G. Nenciu, Rev. Mod. Phys. 63, 91 (1991).

[4] S. de Gironcoli, S. Baroni, and R. Resta, Phys. Rev. Lett. 62, 2853 (1989); P. Giannozzi et al., Phys. Rev. B 43, 7231 (1991); X. Gonze, Phys. Rev. B 55, 10337 (1997).

[5] A. Dal Corso, F. Mauri, and A. Rubio, Phys. Rev. B 53, 15638 (1996).

[6] A. Putrino, D. Sebastiani, and M. Parrinello, J. Chem. Phys. 113, 7102 (2000).

[7] R. W. Nunes and X. Gonze, Phys. Rev. B 63, 155107 (2001).

[8] N. Sai, K. Rabe, and D. Vanderbilt, cond-mat/0205442.

[9] C. Zener, Proc. Roy. Soc. (London) 145, 523 (1934).

[10] Charge leakage also occurs in systems with surfaces (fieldinduced autoionization). However, it is easily avoided by using a standard localized-orbital basis that spans only the region where the system is located, which prevents tunneling into distant regions. In solid-state physics, on the other hand, such distant regions must be covered by basis functions because they are part of the sample.

[11] K. Kunc and R. Resta, Phys. Rev. Lett. 51, 686 (1983).

[12] R. D. King-Smith and D. Vanderbilt, Phys. Rev. B 47, 1651 (1993); D. Vanderbilt and R. D. King-Smith, Phys. Rev. B 48, 4442 (1993). Although the original derivation assumed shorted boundary conditions, we have shown that the same formula remains valid for $\mathcal{E} \neq 0$ [15].

[13] R. W. Nunes and D. Vanderbilt, Phys. Rev. Lett. 73, 712 (1994).

[14] P. Fernandez, A. Dal Corso, and A. Baldereschi, Phys. Rev. B 58, R7480 (1998).

[15] I. Souza, J. Íñiguez, and D. Vanderbilt (unpublished).

[16] W. Kohn, Phys. Rev. 133, A171 (1964).

[17] I. Souza, T. Wilkens, and R. M. Martin, Phys. Rev. B 62, 1666 (2000).

[18] J. B. Krieger and G. J. Iafrate, Phys. Rev. B 33, 5494 (1986); R. Gebauer and R. Car (unpublished).

[19] M. C. Payne et al., Rev. Mod. Phys. 64, 1045 (1992).

[20] D. Vanderbilt, J. Phys. Chem. Solids 61, 147 (2000).

[21] D. F. Nelson, Electric, Optic, and Acoustic Interactions in Dielectrics (Wiley, New York, 1979).

[22] The ABINIT code is a common project of the Université Catholique de Louvain, Corning Incorporated, and other contributors (URL http://www.abinit.org).

[23] J. Singh, Physics of Semiconductors and Their Heterostructures (McGraw-Hill, New York, 1993).

[24] G. Lucovsky, R. M. Martin, and E. Burstein, Phys. Rev. B 4, 1367 (1971). 